\title{
The nuclear radio-optical properties of intermediate-redshift FR II radio galaxies and quasars ${ }^{\star}$
}

\author{
S. Varano ${ }^{1,2, \star \star}$, M. Chiaberge ${ }^{1}$, F. D. Macchetto ${ }^{2, \star \star \star}$, and A. Capetti ${ }^{3}$ \\ 1 Istituto di Radioastronomia del CNR, Via P. Gobetti 101, Bologna 40129, Italy \\ e-mail: varano_s@ira.cnr.it \\ 2 Space Telescope Science Institute, 3700 San Martin Dr., Baltimore, MD 21210, USA \\ 3 INAF - Osservatorio Astronomico di Torino, Strada Osservatorio 20, Pino Torinese 10025, Italy
}

\section{Received 19 February 2004 / Accepted 15 July 2004}

\begin{abstract}
We extend the study of the nuclei of 3CR objects as seen with the HST to higher-redshift FR II radio sources $(0.4<z<0.6)$. Our results reflect what has been found for FR II of lower redshift. The position of the nuclei in the plane formed by optical and radio luminosities is related to their optical spectral classification: the nuclei of both high and low excitation galaxies lie on the correlation found for FR I sources, while broad-line objects have a significant optical excess. The nuclear properties of these sources are better understood when we consider the equivalent widths of the [OIII] emission line with respect to their optical luminosities. Even in the range of redshift considered here, low excitation galaxies show peculiar nuclear properties, more similar to those observed in FR I. This confirms that not all narrow-line FR II are unified with quasars. Our findings have important implications for the FR II-quasar unification scheme: by reconsidering the classification of all $3 \mathrm{CR}$ radio galaxies with $z<1$ in the light of their nuclear properties, we derive the opening angle of the obscuring torus for different redshift bins. We find that the covering factor of the obscuring structure decreases as the redshift increases $\left(\theta \sim 44^{\circ}\right.$ for $z<0.3$ to $\theta \sim 56^{\circ}$ for $0.5<z<1$ ). We argue that this behavior may be interpreted in the framework of the receding torus model, in which the opening angle of the torus increases as the luminosity of the accretion disk around the central black hole increases.
\end{abstract}

Key words. galaxies: active - galaxies: nuclei - quasars: general

\section{Introduction}

In the framework of the radio-loud AGN unification scheme, Fanaroff \& Riley I and II (FR I and FR II) radio sources (Fanaroff \& Riley 1974) are believed to be the parent population of BL Lacs and QSO, respectively (see Urry \& Padovani 1995, for a review). In this scenario, the differences between the observed emission properties are ascribed to a different orientation of the anisotropic central emission. There are two main reasons for anisotropy: the presence of non-thermal emission from a relativistic jet, which dominates the radiation observed in BL Lacs and Flat Spectrum Radio Quasars, and the presence of an obscuring "torus", which prevents us from observing directly the central parsecs in objects observed along lines of sight perpendicular to the jet axis.

Optical studies of radio galaxies are particularly important not only for investigating their large-scale properties, such

\footnotetext{
* Based on observation obtained at the Space Telescope Science Institute, which is operated by the Association of Universities for Research in Astronomy, Incorporated, under NASA contract NAS 5-26555.

$\star \star$ Visiting student, Summer Student Program 2002.

$\star \star \star$ Space Telescope Division, ESA.
}

as the structure of the host and the environment (see, e.g., Zirbel 1996, 1997), but also for exploring the physical conditions of the central regions. The high resolution and sensitivity provided by HST optical images allow us to separate the AGN emission from the stellar host galaxy background even in the case of low power radio galaxies (FR I).

Chiaberge et al. (1999) (hereafter, Paper I) and Chiaberge et al. (2002) (hereafter, Paper II) have studied complete samples of FR I and FR II radio galaxies with $z<0.3$ using HST images taken as part of the 3CR snapshot surveys (e.g., Martel et al. 1999; De Koff et al. 1996). They analyzed the properties of unresolved nuclei which have been found to be present in the great majority of the objects. The optical nuclei of FR I correlate linearly with the radio nuclear emission over four orders of magnitude, both in flux and luminosity. In analogy with the origin of the radio cores, the CCC (central compact core) emission has been interpreted as nonthermal synchrotron radiation from the base of the relativistic jet. Furthermore, the high detection rate $(85 \%)$ suggests that we have a direct view of the central regions in most objects, and that a geometrically and optically thick obscuring torus is generally not present in FR I objects. Thus, FR I intrinsically lack strong broad emission lines. 
FR II with redshift $z<0.3$ show a more complex behavior, although their properties are clearly related to their optical spectral classification. Objects with broad emission lines (broad-line radio galaxies and quasars) have bright nuclei which show an optical excess with respect to the FR I correlation, probably because of the dominance of thermal emission from the accretion disk in the optical band. Sources in which only narrow lines are present are distinguished into high and low excitation galaxies (HEG and LEG - Laing et al. 1994). Approximately 50\% of the HEG do not show any nuclear optical component and they are interpreted as obscured sources, as expected in the framework of the unification scheme. On the other hand, the nuclei of HEG, when present, do lie on the correlation (or slightly above it). This behavior is unexpected, and their nature is intriguing. However, by including the nuclear Equivalent Width of the [OIII] emission line as a further parameter in the diagnostic diagrams we can discriminate between nuclei observed directly and those that are obscured and seen only through scattered emission (Paper II).

LEG seem to behave differently from other radio galaxies with FR II morphology. A significant fraction of them have faint optical nuclei similar to those of FR I and they lie on the radio-optical correlation. Their nuclear physical properties are probably more similar to those of FR I, and this appears to be in contrast with the "standard" zeroth-order unification model. These sources might be associated with those BL Lacs that show radio morphologies and total radio power more typical of FR II sources, thus providing support for a revised unification scenario (Laing et al. 1994; Jackson \& Wall 1999; Hardcastle et al. 2004).

The limitation so far has been that the results described above hold for low redshift sources. The aim of this paper is to extend the previous studies to a sample of higher redshift FR II, in the range $0.4<z<0.6$. This is particularly important for two reasons: i) the radio-loud AGN unification scheme was originally proposed by Barthel et al. (1989) using a sample of high redshift $(0.5<z<1) 3 \mathrm{CR}$ objects; ii) cosmological evolution may play a role in changing the structure of radio galaxies and quasar. Therefore, the conclusions drawn for the low redshift sample may not apply to high redshift objects, and the discrepancies with the unification scheme found in the analysis of nearby samples may be explained in terms of cosmological evolution. Instead, if the same behavior is still observed as redshift increases, the unification scheme needs to be revised.

The paper is organized as follows: in Sect. 2 we describe our sample of FR II and in Sect. 3 the HST observations; in Sect. 4 we present the results of the photometry of the nuclei; in Sect. 5 we discuss our results and consider their implications for the unification models. In Sect. 6 we summarize the main results, draw conclusions and discuss future perspectives. $H_{0}=$ $75 \mathrm{~km} \mathrm{~s}^{-1} \mathrm{Mpc}^{-1}$ and $q_{0}=0.5$ are used throughout the paper.

\section{The sample}

The sample considered in this paper comprises all radio galaxies belonging to the 3CR catalog (Spinrad et al. 1985) with redshifts in the range $0.4<z<0.6$, morphologically classified as FR II.
We checked the morphological classification, optical identification, and redshift of all sources by searching the literature for the most recent data. We exclude 3C 119 from our sample since its redshift is $z=1.023$ (Eracleous \& Halpern 1994) instead of $z=0.408$, as originally reported in the 3CR catalog. The final list is thus a complete, flux and redshift limited sample of 28 FR II radio sources (Table 1). Note that, to the best of our knowledge, for 3C 306.1 and 3C 275 no information on the radio core is available .

We classify our sources on the basis of their emission line properties, adopting the scheme defined by Jackson \& Rawlings (1997). Weak Quasars (WQ) and quasars (QSO) are objects for which at least one broad line has been observed. These two classes differ only in optical continuum luminosity: $L_{5500 \AA}>10^{23} \mathrm{~W} \mathrm{~Hz}^{-1}$ for QSO, while WQ are fainter. Thus, in the following, we refer to them as a single population of broadline objects (BLO). High and low excitation galaxies (HEG and LEG) are narrow-line sources, and are classified on the basis of their $[\mathrm{OII}] /[\mathrm{OIII}]$ ratio (>1 for a LEG) and/or [OIII] equivalent width (less than $10 \AA$ for a LEG).

In Table 1 we report radio and optical data for the complete sample as taken from the literature.

\section{HST observations}

Optical HST observations are available in the public archive for all but one (namely 3C 327.1) of the sources of our sample. The HST images were taken using the Wide Field and Planetary Camera 2 (WFPC2). The projected pixel sizes of the Planetary Camera (PC) and the Wide Field cameras (WF) are 0.0455 arcsec and 0.1 arcsec, respectively. The three WF cameras cover a "L" shaped field of view of 150 " $\times 150$ ", while the PC covers an area of $35^{\prime \prime} \times 35^{\prime \prime}$.

Twenty-two sources were observed with the $F 702 \mathrm{~W}$ filter as part of the HST snapshot survey of 3CR radio sources (GO 5476, Lehnert et al. 1999; De Koff et al. 1996). 3C 295 was observed with the $F 702 \mathrm{~W}$ filter as part of a different program (GO 5378), while 3C 275.1 and 3C 345 were observed with the $F 675 W$ and $F 555 W$, respectively. Deep observations with the $F 702 \mathrm{~W}$ filter are also available for 3C 16 and 3C 435 from GO 6675. Further observations with the $F 785 L P$ filter (taken as part of program GO 9045) are available for eleven radio galaxies of our sample. However, although the $F 785 \mathrm{LP}$ filter has a wide passband $(\sim 2000 \AA)$, and deep observations were obtained $\left(t_{\exp }=2000 \mathrm{~s}\right)$, the target is always located in the WF3 Camera. Therefore, since our goal is to check for the presence of unresolved nuclear components, we favor observations in which the target lies in the PC, which has a smaller projected pixel size, thus providing a better sampling of the PSF. Since 3C 327.1 has not been observed with HST, our final sample is composed of twenty-seven sources. In Table 2 we summarize the details of the HST observations.

The data have been processed through the standard OTFR system (On-The-Fly Reprocessing) pipeline for data processing and calibration (Baggett et al. 2002).

For twelve sources the observations have been split into multiple exposures to remove cosmic ray events. We use the IRAF task CRREJ which combines the images and rejects 
Table 1. Summary of data from the literature for our sample. The spectral classification is from Willott's catalog at the URL http://www-astro.physics.ox.ac.uk/ cjw/3crr/3crr.html, except $\left({ }^{a}\right)$ for which we used the classification published in Jackson \& Rawlings (1997) (spectra by Jackson \& Browne 1991, for 3C 345, Tadhunter et al. 1993, for 3C 327.1 and Spinrad et al. 1985, for the rest) and $\left(^{b}\right)$ for which we report the classification available in the NASA/IPAC Extragalactic Database (no references for the spectra).

\begin{tabular}{|c|c|c|c|c|c|}
\hline $\begin{array}{l}\begin{array}{l}\text { Source } \\
\text { name }\end{array} \\
\end{array}$ & $\begin{array}{l}\text { Redshift } \\
z\end{array}$ & $\begin{array}{r}\log L_{\mathrm{r}}(5 \mathrm{GHz}) \\
\operatorname{erg~s}^{-1} \mathrm{~Hz}^{-1}\end{array}$ & $\begin{array}{c}\log L_{\text {tot }}(178 \mathrm{MHz}) \\
\operatorname{erg~s}^{-1} \mathrm{~Hz}^{-1}\end{array}$ & $\begin{array}{c}\log L_{\text {[OIII] }} \\
\mathrm{W}\end{array}$ & $\begin{array}{l}\text { Spectral } \\
\text { class. }\end{array}$ \\
\hline $3 \mathrm{C} 16$ & 0.405 & $<30.45$ & 34.77 & - & HEG \\
\hline $3 \mathrm{C} 19$ & 0.482 & $<33.22$ & 35.00 & - & LEG \\
\hline $3 \mathrm{C} 46$ & 0.4373 & 31.17 & 34.82 & 36.04 & HEG \\
\hline $3 \mathrm{C} 47$ & 0.425 & 32.65 & 35.20 & 36.52 & QSO \\
\hline $3 C 99$ & 0.426 & 32.99 & 34.81 & - & $\mathrm{HEG}^{b}$ \\
\hline 3C 147 & 0.545 & 34.22 & 35.25 & 37.03 & QSO \\
\hline $3 C 154$ & 0.5804 & 33.86 & 35.49 & - & $\mathrm{QSO}^{b}$ \\
\hline $3 \mathrm{C} 172$ & 0.5191 & $<31.70$ & 35.18 & - & HEG \\
\hline $3 C 200$ & 0.458 & 32.41 & 35.01 & - & LEG \\
\hline $3 C 215$ & 0.411 & 31.96 & 34.80 & 35.83 & QSO \\
\hline $3 \mathrm{C} 225.0 \mathrm{~B}$ & 0.58 & $<31.12$ & 35.45 & - & HEG? \\
\hline $3 C 228$ & 0.5524 & 32.19 & 35.32 & - & HEG \\
\hline $3 \mathrm{C} 244.1$ & 0.428 & $<30.56$ & 35.13 & 36.27 & HEG \\
\hline $3 \mathrm{C} 274.1$ & 0.422 & 31.60 & 34.99 & 34.60 & $\operatorname{HEG}\left(\mathrm{LEG}^{*}\right)$ \\
\hline $3 \mathrm{C} 275$ & 0.48 & - & 35.09 & 36.26 & $\mathrm{LEG}^{a}$ \\
\hline $3 \mathrm{C} 275.1$ & 0.557 & 33.37 & 35.34 & 35.81 & QSO \\
\hline $3 C 295$ & 0.4614 & 31.71 & 34.79 & 35.23 & LEG \\
\hline 3C 306.1 & 0.441 & - & 35.95 & - & $\mathrm{HEG}^{a}$ \\
\hline $3 C 313$ & 0.461 & $<30.87$ & 35.17 & 35.71 & $\mathrm{HEG}^{a}$ \\
\hline 3C 327.1 & 0.4628 & 32.68 & 35.24 & 35.95 & $\mathrm{HEG}^{a}$ \\
\hline $3 C 330$ & 0.55 & 30.93 & 35.51 & - & HEG \\
\hline 3C 334 & 0.5551 & 33.12 & 35.11 & 36.61 & QSO \\
\hline 3C 341 & 0.448 & 30.84 & 34.87 & 36.04 & HEG \\
\hline $3 C 345$ & 0.594 & 34.87 & 35.23 & 36.17 & $\mathrm{QSO}^{a}$ \\
\hline $3 \mathrm{C} 411$ & 0.467 & 32.53 & 35.10 & - & $\mathrm{HEG}^{a}$ \\
\hline 3C 427.1 & 0.572 & 30.57 & 35.53 & - & LEG \\
\hline $3 \mathrm{C} 435 \mathrm{~A}$ & 0.471 & 32.12 & 34.95 & - & Unclass \\
\hline $3 \mathrm{C} 455$ & 0.543 & 31.19 & 35.19 & 36.31 & WQ \\
\hline
\end{tabular}

* 3C 274.1 is classified by Willott as a HEG, but Jackson \& Rawlings classify it as a doubtful LEG. See discussion in Sect. 5.1.

cosmic rays through an iterative process. For fifteen out of the twenty-three sources observed with the $F 702 \mathrm{~W}$ filter multiple exposures are not available. In these cases, we carefully checked the central regions of the target to ensure that the area of interest is not contaminated by cosmic rays. Cosmic rays are present in the nuclear regions of the F702W images of 3C 19 , 3C 274.1 and 3C 330. Therefore, for such objects we used observations with different filters (see Table 2). For 3C 275, a cosmic ray affects the central region of the only HST image available, preventing any estimate of the nuclear emission.

\section{Identification and photometry of central compact cores}

The identification of an unresolved nuclear component is not straightforward for sources in the redshift range considered here. Typically, the diameter of the host galaxy does not exceed $\sim 10$ pixels and a steep radial brightness profile might be confused with a nuclear compact component. Thus, a careful inspection of the galaxy's profile is needed.

We use the IRAF RADPROF task on a region of 10 pixels from each galaxy center to derive its radial brightness profile. A steepening of the brightness profile occurring in the central pixels, which results in an $F W H M$ comparable to that of an unresolved source $(\sim 0.08$ arcsec $)$ is indicative of the presence of a central compact core.

In twelve out of the twenty-seven sources ( $44 \%$ of the sample) the $F W H M$ of the central region is $<0.08$ arcsec. To measure the flux of the CCC we performed aperture photometry. The highest source of error is the determination of the background: we set the galaxy background level at a distance of four or five pixels from the center of the galaxy, for the objects with $0.5<z<0.6$ and $0.4<z<0.5$, respectively.

In seven galaxies we measured an FWHM of the central region larger than $0.1 \mathrm{arcsec}$. For these objects we estimated an upper limit to the nuclear source by measuring the light excess in the central $3 \times 3$ pixels with respect to the surrounding galaxy background. For sources in the redshift range $0.5<z<0.6$ we lowered the central box size down to $2 \times 2$ pixels.

Four objects, namely 3C 16, 3C 46, 3C 99 and 3C 313, show a complex morphology which prevents us from detecting any nuclear emission. Note that the optical counterpart of 3C 313 was previously identified on the HST image as a bright (quasar-like) point source (De Koff et al. 1996). Its radio core has been only marginally detected in a VLA observation 
Table 2. Log of HST observations.

\begin{tabular}{lccc}
\hline \hline Source name & Filter & $t_{\text {exp }}(\mathrm{s})$ & GO program ID \\
\hline 3C 16 & $F 702 W$ & 8300 & 6675 \\
3C 19 & $F 785 L P$ & 1000 & 9045 \\
3C 46 & $F 702 W$ & 300 & 5476 \\
3C 47 & $F 702 W$ & 280 & 5476 \\
3C 99 & $F 702 W$ & 300 & 5476 \\
3C 147 & $F 702 W$ & 280 & 5476 \\
3C 154 & $F 702 W$ & 280 & 5476 \\
3C 172 & $F 702 W$ & 300 & 5476 \\
3C 200 & $F 702 W$ & 300 & 5476 \\
3C 215 & $F 702 W$ & 280 & 5476 \\
3C 225.0B & $F 702 W$ & 300 & 5476 \\
3C 228 & $F 702 W$ & 300 & 5476 \\
3C 244.1 & $F 702 W$ & 300 & 5476 \\
3C 274.1 & $F 785 L P$ & 2000 & 9045 \\
3C 275 & $F 702 W$ & 300 & 5476 \\
3C 275.1 & $F 675 W$ & 1000 & 5978 \\
3C 295 & $F 702 W$ & 6300 & 5378 \\
3C 306.1 & $F 702 W$ & 300 & 5476 \\
3C 313 & $F 702 W$ & 300 & 5476 \\
3C 330 & $F 555 W$ & 600 & 6348 \\
3C 334 & $F 702 W$ & 280 & 5476 \\
3C 341 & $F 702 W$ & 300 & 5476 \\
3C 345 & $F 555 W$ & 2800 & 5235 \\
3C 411 & $F 702 W$ & 300 & 5476 \\
3C 427.1 & $F 702 W$ & 300 & 5476 \\
3C 435A & $F 702 W$ & 7400 & 6675 \\
3C 455 & $F 702 W$ & 300 & 5476 \\
\hline
\end{tabular}

at $5 \mathrm{GHz}$ (Baum et al. 1988), but it is clearly seen at $8 \mathrm{GHz}$ (Bogers et al. 1994). The position of the core, as estimated from the radio map, corresponds to a faint galaxy on the HST image, which is located close to the edge of the PC field (Fig. 1). This object, which we consider as the true host galaxy of 3C 313, is located $\sim 5$ arcsec away from the previous identification in the HST image by De Koff et al. (1996). Unfortunately, as reported above, the host galaxy of 3C 313 has a close companion and shows a complex nucleus, with possible dust lanes obscuring the central region.

A further problem in estimating the nuclear continuum is represented by contamination from emission lines, which may be significant especially in the case of BLO and HEG. In Paper II we considered the contamination from broad $\mathrm{H} \alpha$ lines in BLO. We showed that, for the range of redshifts in which the line falls in the filter passband, the flux of the emission line represents only 10-20\% of the nuclear flux. For the range of redshifts and for the filters considered in this paper, the emission lines that may contribute to the nuclear flux observed in the broad band filter images are mainly represented by the [OIII]5007 and H $\beta$. For the HEG 3C 244.1 an HST image taken with a narrow band filter (FR680N) centered on [OIII]5007 line is available in the public archive. It is well known that a significant fraction of the emission line flux in radio galaxies is produced in extended (kpc-scale) regions of the host (e.g., Baum et al. 1988). For 3C 244.1, we estimate that the [OIII] nuclear flux corresponds to $\sim 50 \%$ of the total [OIII] flux derived from ground based observations (Jackson \& Rawlings 1997). We

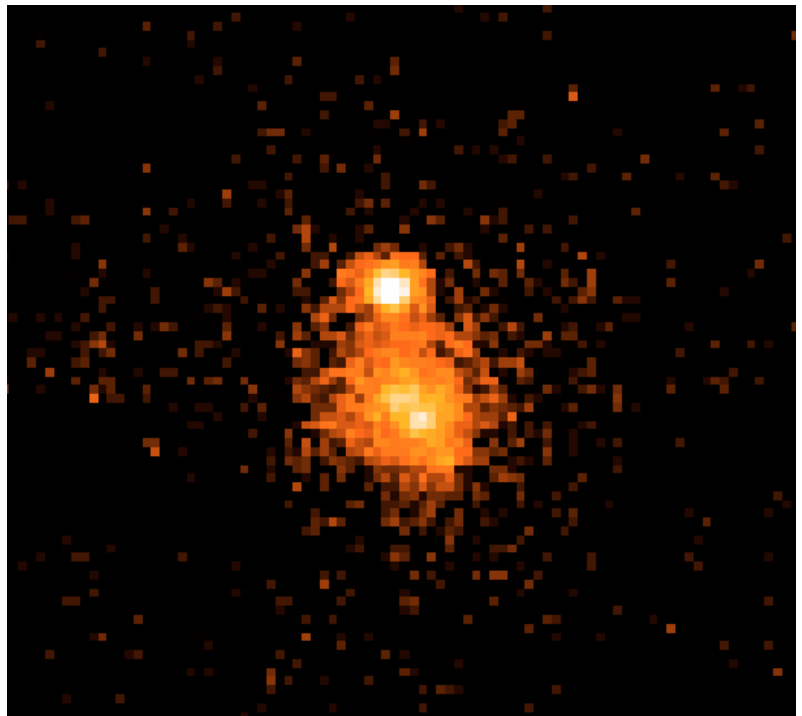

Fig. 1. HST image of the host galaxy of 3C 313 (in the center). The size of the image is $4 \times 4 \operatorname{arcsec}^{2}$. The nucleus of this source has a complex morphology (see discussion in the text, Sect. 4).

measured the nuclear [OIII] flux from the narrow band image, and we found that its contribution to the total count-rate observed in the broad-band image is $\sim 50 \%$ of the nuclear flux. Although this appears to be a substantial contribution, it does not significantly affect our results. For two more HEG (namely 3C 274.1 and 3C 341) the total [OIII] flux is available from the literature, but their nuclei are not detected in our HST images. If we assume that $\sim 50 \%$ of the total [OIII] flux is produced in the nucleus, this would result in a count-rate higher than our detection threshold of $\sim 1.5 \times 10^{-29} \mathrm{erg} \mathrm{cm}^{-2} \mathrm{~s}^{-1} \mathrm{~Hz}^{-1}$, as set by the upper limits we estimate for these objects. This implies that most $(>50 \%)$ of the [OIII] flux is produced in extended (kpc-scale) regions of the galaxy, and thus our estimates for the nuclear continuum are suitable for the purpose of the work presented here. However, note that even if the three CCC of the HEG were entirely due to line emission, our results are not substantially affected by substituting detections with upper limits, since their representative points in the diagnostic diagrams would point to the regions were we expect the HEG to be located.

The results of our search for CCC are summarized in Tables 3 and 4.

\section{Results and discussion}

To study the nature of the nuclei we take advantage of the results obtained for the lower redshift samples $(z<0.3)$ in Papers I and II. In Fig. 2 we plot our sources in the plane defined by the CCC luminosity vs the radio core luminosity and compare their behavior to that of the nearby $3 \mathrm{C}$ galaxies. Note that, due to the small redshift range of our sample, the distributions in either flux or luminosity do not differ significantly.

The positions of our sources in the luminosity plot with respect to the FRI correlation are related to their optical spectral classifications (Fig. 3). Their behavior is similar to that of low redshift FR II sources, but shifted toward higher luminosities. 


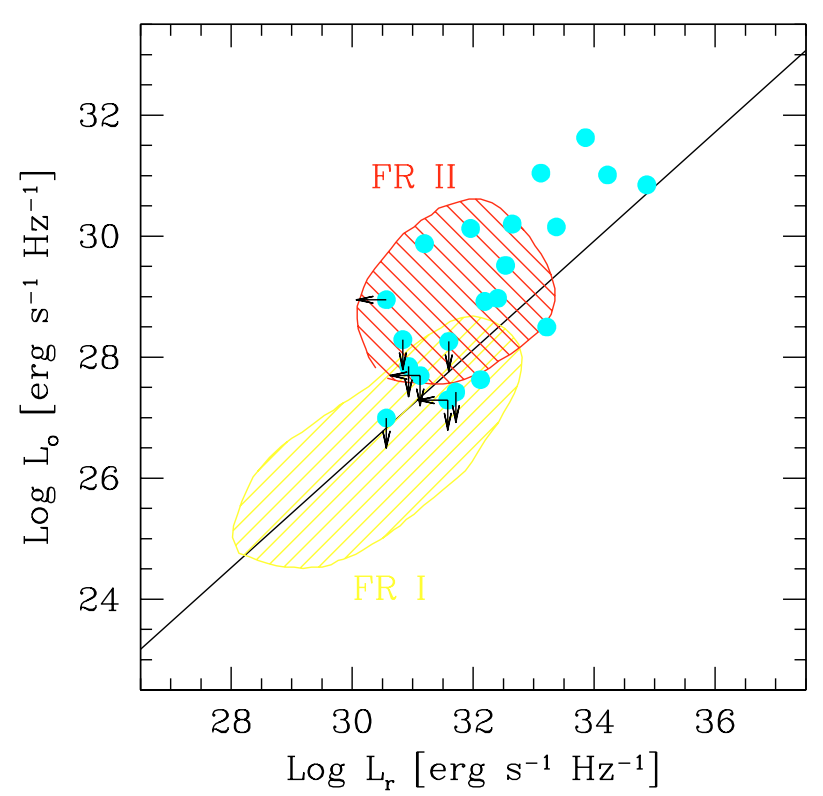

Fig. 2. Optical luminosity of the CCC vs. radio core luminosity. The two shaded regions represent the positions of the FR I sample and the sample of FR II at $z<0.3$.

Table 3. Result from identification and photometry of central compact cores in our sample.

\begin{tabular}{|c|c|c|c|}
\hline $\begin{array}{l}\text { Source } \\
\text { name }\end{array}$ & $\begin{array}{r}\log L_{\mathrm{o}}(7000 \AA) \\
\mathrm{erg} \mathrm{s}^{-1} \mathrm{~Hz}^{-1}\end{array}$ & $\begin{array}{l}\text { Source } \\
\text { name }\end{array}$ & $\begin{array}{r}\log L_{\mathrm{o}}(7000 \AA) \\
\operatorname{erg~s}^{-1} \mathrm{~Hz}^{-1}\end{array}$ \\
\hline $3 C 16$ & Complex & $3 C 275$ & $\mathrm{CR}$ \\
\hline $3 \mathrm{C} 19$ & $<28.50$ & 3C 275.1 & 30.15 \\
\hline $3 \mathrm{C} 46$ & Complex & $3 C 295$ & $<27.67$ \\
\hline $3 \mathrm{C} 47$ & 30.20 & 3C 306.1 & $<28.20$ \\
\hline 3C 99 & Complex & $3 C 313$ & Complex \\
\hline 3C 147 & 31.01 & 3C 327.1 & Not obs. \\
\hline 3C 154 & 30.93 & $3 C 330$ & $<27.85$ \\
\hline 3C 172 & $<27.29$ & 3C 334 & 31.04 \\
\hline $3 \mathrm{C} 200$ & 28.97 & $3 C 341$ & $<28.29$ \\
\hline $3 \mathrm{C} 215$ & 30.13 & $3 C 345$ & 30.85 \\
\hline $3 \mathrm{C} 225.0 \mathrm{~B}$ & $<27.69$ & 3C 411 & 29.52 \\
\hline $3 C 228$ & 28.92 & $3 \mathrm{C} 427.1$ & $<26.99$ \\
\hline 3C 244.1 & 28.95 & $3 \mathrm{C} 435 \mathrm{~A}$ & $<27.63$ \\
\hline $3 \mathrm{C} 274.1$ & $<28.26$ & $3 C 455$ & 29.88 \\
\hline
\end{tabular}

Broad Line Objects are the brightest, both in the optical and radio band. They typically show an optical excess of 1-2 dex with respect to the FR I correlation. As discussed in Paper II, this optical excess can be explained if the optical emission is dominated by thermal radiation from the accretion disk. However, one source (3C 345) lies on the radio-optical correlation. This is not surprising, since 3C 345 is a Flat Spectrum Radio Quasar and both its radio and optical nuclear emission are dominated by non-thermal synchrotron radiation (Moore \& Stockman 1981). Note that the apparent correlation between $L_{\mathrm{o}}$ and $L_{\mathrm{r}}$ for BLO is not real and only due to the common dependence on redshift of the two quantities. In fact, we find that no

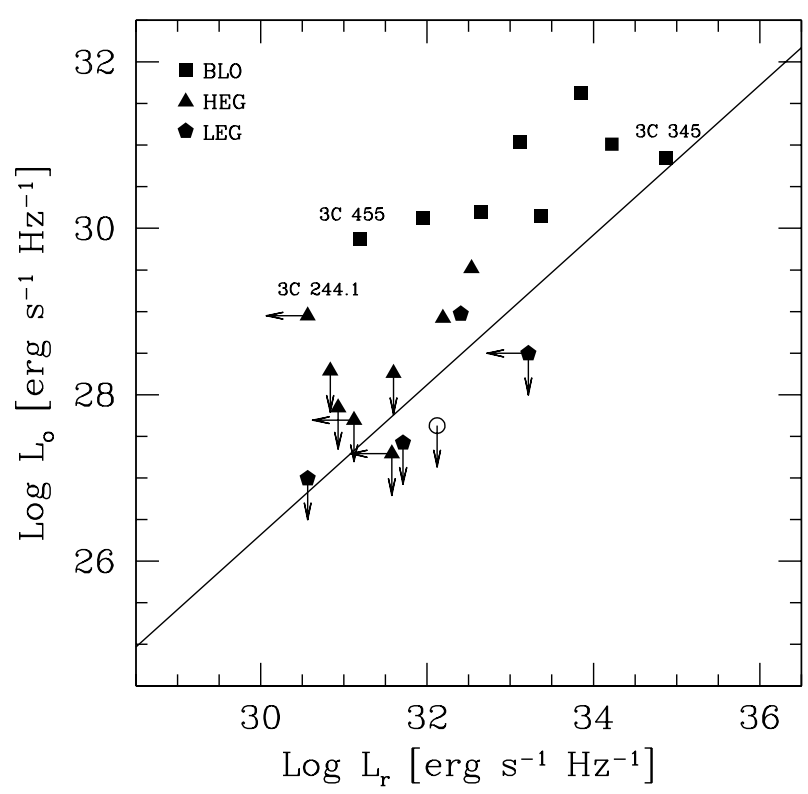

Fig. 3. Same as Fig. 2; enlargement of the range of luminosities of the present sample. Different symbols refer to different spectral classifications. The empty circle corresponds to the only unclassified source (namely 3C 435A).

correlation is present when fluxes instead of luminosities are considered.

The nuclei of the LEG lie very close to the FR I correlation. Although we only have three sources (one CCC and two upper limits), their behavior is similar to that of low- $z$ LEG.

The HEG show a more complex behavior. Six out of seven lie very close to the FR I regression line (within $2 \sigma$ ). However, the location of 3C 244.1 is puzzling. Its representative point lies more than 2 dex away from the correlation. Its optical core is clearly identified in the HST image but only an upper limit to the radio core flux is available. We will discuss in detail the nature of this object in the following section.

As shown in Paper II, the position of HEG nuclei in the radio-optical plane can be misleading. A significant fraction of low-redshift HEG lie on the FR I correlation "by chance" and the nature of their optical emission can be assessed only when a further parameter is included, i.e. the equivalent width of the [OIII] emission line. Therefore, before drawing conclusions on the nature of high redshift FR II nuclei, we discuss the properties of our sources as far as their emission lines are concerned.

\subsection{Equivalent width of the [OIII] emission line vs. the radio-to-optical flux ratio}

In Fig. 4 we plot the ratio between the [OIII] emission line flux and the optical continuum (which represents the "nuclear" Equivalent Width of [OIII]) vs the optical-to-radio core ratio. The position of the sources along the $Y$ axis indicates whether the nucleus is observed directly or is obscured to our line of sight and visible only through scattered light. In fact, in the scenario in which the narrow line region is photo-ionized by UV radiation produced in the central accretion disk, the strength of the emission lines (in particular those of high 
Table 4. Summary of the optical properties of the sources at $0.4<z<0.6$ observed with HST and results of our study on the images. Note that only one source of our sample (classified as HEG) has no HST data available (3C 327.1). 3C 275 has also been excluded because of a cosmic ray event in the central region of the HST image (see text, Sect.3).

\begin{tabular}{lccccc}
\hline \hline & CCC detections & Optical upper limits & Complex morphology & TOT & \% CCC detections \\
\hline BLO & 8 & - & - & 8 & $100 \%$ \\
LEG & 1 & 3 & 1 & 5 & $20 \%$ \\
HEG & 3 & 6 & 4 & 13 & $21 \%$ \\
\hline All sources & 12 & 9 & 5 & 26 & $44 \%$ \\
\hline
\end{tabular}

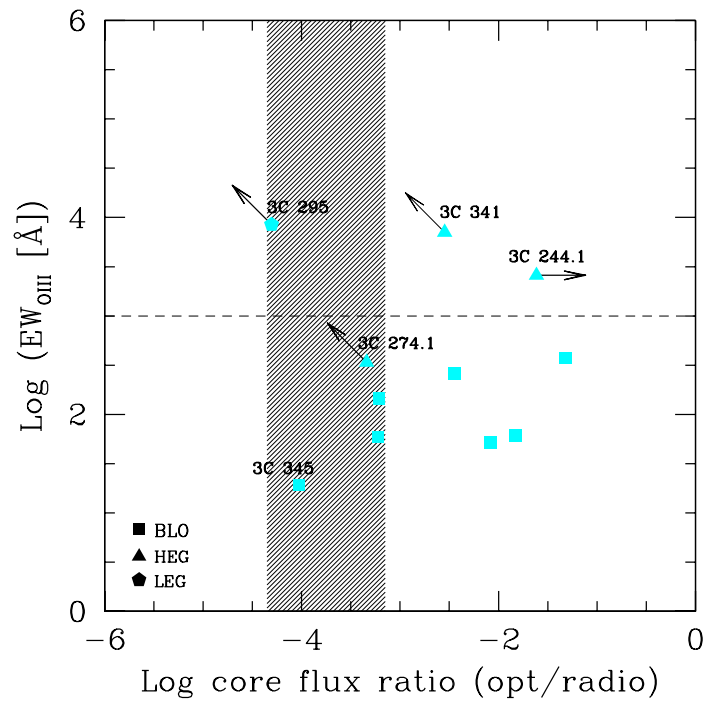

Fig. 4. Equivalent Width of the [OIII] emission line, measured with respect to the CCC emission, plotted vs. the ratio between the optical CCC to radio core flux. The shaded area represents the dispersion $(1 \sigma)$ of the linear correlation between radio core and CCC luminosity found for FR I in Paper I. The dashed horizontal line is the separation between "obscured" and "unobscured" sources (see text, Sect. 5.1).

ionization levels) is closely correlated with the intensity of the nuclear source. If we have a clear view of the central regions of the galaxy, a low $E W$ of the [OIII] emission line is expected. Conversely, a high $E W(\log E W>3.5)$ is measured if the nuclear (ionizing) component is obscured to our line of sight, and only a small fraction of it is seen through scattered light (see Paper II for an extensive discussion).

The location of the representative points along the $X$ axis reflects the distribution observed in the plane formed by the optical vs. radio core emission. The shaded region represents the correlation between these two quantities found for FR I radio sources ( $1 \sigma$ dispersion). Objects lying on the right side of this region have an optical excess with respect to the correlation. In the nearby sample, BLO are found in the lower right region of the plane, while the HEG typically show high $E W$ and are interpreted as obscured BLO. Low- $z$ LEG, all of which lie among the FR I in all diagnostic planes, are interpreted as "FRI-like" nuclei, and they do not show evidence for substantial obscuration.

Unfortunately, to the best of our knowledge there are only eleven sources of our sample for which there is optical spectral information in the literature. Three of them are HEG, one is a LEG and seven are BLO.
Table 5. Fraction of BLO, HEG and LEG for different redshift bins The low redshift bin corresponds to the sample of Paper II, the intermediate to this paper, and the high- $z$ bin corresponds to the sample of Barthel (1989).

\begin{tabular}{lccc}
\hline \hline & $z<0.3$ & $0.4<z<0.6$ & $0.5<z<1$ \\
\hline BLO & $20 \%$ & $28 \%$ & $44 \%$ \\
HEG & $46 \%$ & $50 \%$ & $53 \%$ \\
LEG & $25 \%$ & $18 \%$ & $2 \%$ \\
Unclass. & $9 \%$ & $4 \%$ & $1 \%$ \\
\hline
\end{tabular}

All of the BLO lie in the lower-right region of the plane in Fig. 4 and have $E W_{\text {[OIII] }} \sim 100$. This is a signature that we are observing the source of ionization directly. Note that, as already mentioned above, 3C 345 is an outlier because of the dominance of non-thermal emission in the optical band. In the plane of Fig. 4 this has two effects: i) it drives the object toward the FR I "synchrotron" correlation and; ii) it lowers the equivalent width of [OIII], as the emission lines are diluted by the strong non-thermal continuum.

Two of the three HEG (namely 3C 341 and 3C 244.1) have a high $E W[\mathrm{OIII}]$, thus they are similar to the vast majority of low redshift HEG, which lie in the top (left) part of the diagram. 3C 244.1 not only shows an optical excess, as already pointed out in the previous section, but it also has a high value of $E W[\mathrm{OIII}]$. Although the non-detection of its radio core is puzzling, its behavior appears to be similar to that of 3C 184.1 at $z=0.118$ (Paper II). Thus we argue that the location of 3C 244.1 in the diagnostic planes can be explained if this object has an unusually high intrinsic optical-to-radio core flux ratio and its "quasar" nucleus is moderately absorbed, so that its optical broad emission lines are not detected. Alternatively, this object may be a "normal" HEG with a scattered nuclear continuum (but still with a rather unusual optical-to-radio core flux ratio). To test these hypothesis, both deeper radio data and better spectral information are needed: to confirm the former hypothesis we should search for faint broad emission lines in direct light, while for the latter we expect broad emission lines (and nuclear continuum) to be strongly scattered and thus polarized.

3C 274.1 lies among the "unobscured" sources, where LEG are usually found. However, the non-detection of the optical core only sets a limit on its location in this plane. Its [OIII] luminosity $\left(\log L_{[\mathrm{OIII}]}=34.60\right)$ is typical of LEG and significantly smaller than any other HEG in our sample ( $\log L_{[\mathrm{OIII}], \text { med }} \sim 36.37$ ). Therefore, we argue that this source is 

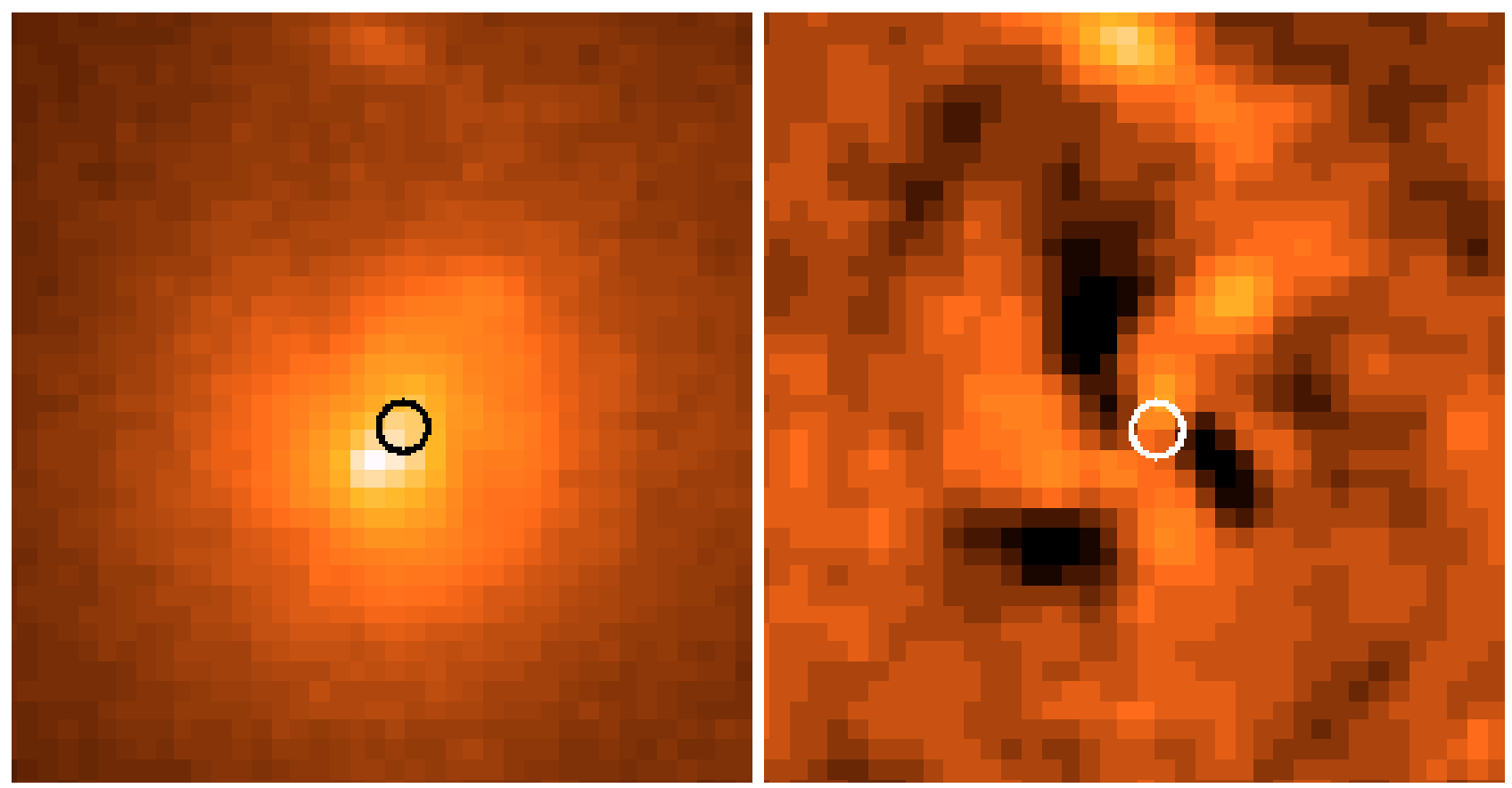

Fig. 5. HST optical image of 3C 295 (on the left) and residuals after the subtraction of the model obtained with a isophotal fitting (on the right). The circle is the center of the galaxy model. The dimension of both boxes is $1.8 \times 1.8 \mathrm{arcsec}^{2}$. See text for discussion (Sect. 5.1).

actually a LEG, as already tentatively proposed by Jackson \& Rawlings (1997).

The only LEG with [OIII] information, 3C 295, lies among the obscured sources, well out of the region occupied by low redshift LEG. As already pointed out above, low redshift LEG are usually found within the FR I region, i.e. on the radiooptical correlation and with low $E W$ [OIII].

The [OIII] luminosity of $3 \mathrm{C} 295\left(\log L_{[\mathrm{OIII}]}=35.23\right)$ is significantly higher than the average of all of the 3 C LEG, and it is similar to that of HEG. This could imply that the source has been misclassified. On the other hand, the optical image (Fig. 5, left panel) reveals that the host galaxy has a complex morphology and presents signs of interaction. To test if the absence of a nuclear source is due to the presence of dust that covers the nuclear regions, we performed isophotal fitting of the galaxy and we subtracted the resulting model from the image. This procedure highlights possible inhomogeneities in the galaxy structure (Fig. 5, right panel). The resulting image confirms that the morphology of the galaxy is complex. There is evidence for absorbing structures, possibly a dust lane, that might lower the emission from the nucleus. Furthermore, the center of the galaxy lies at a distance of $\sim 0.2$ arcsec from the peak of its surface brightness profile (the "nucleus" for our estimate of the optical upper limit). We conclude that because of the complexity of the central region of the galaxy, the upper limit measured on the HST image is probably unreliable, and the $E W([\mathrm{OIII}])$ is in turn overestimated. An $A_{\mathrm{V}} \sim 2-3$ mag is sufficient to hide the central source. In the light of the present data we cannot discriminate between the two scenarios proposed, i.e. misclassification of the source (which would be a HEG) or the presence of a moderate amount of absorption produced by large-scale dust lanes that prevents the detection of the optical nucleus.
Summarizing, the behavior of the high redshift BLO is completely in agreement with that of their low-redshift counterparts. The lack of complete spectral information for both the LEG and HEG in the high- $z$ sample prevents us from drawing any firm conclusion about these sources. However, we have shown that it is plausible that the scenario proposed for nearby objects still fits the behavior of the distant sample: LEG have FR I-like nuclei and HEG are absorbed QSO.

\subsection{Implications for the unified models}

Let us now compare our findings with the behavior of lower redshift FR II radio sources in the framework of the unification models for quasars and narrow-lined radio galaxies (Barthel et al. 1989).

In the range $0.4<z<0.6,28 \%$ of the sources are BLO, $50 \%$ are HEG and $18 \%$ are LEG (only one object is unclassified). Compared with the low redshift sample of Paper II (65 objects, of which $20 \%$ are BLO, $46 \%$ are HEG, $25 \%$ are LEG and $9 \%$ are unclassified) the fraction of BLO increases with redshift while the fraction of LEG decreases. On the other hand, the fraction of HEG appears to be fairly constant. If we translate this result into an estimate for the "opening angle" $\theta$ of the obscuring torus, using $P=1-\cos \theta$ (where $P$ is the fraction of objects with broad lines), for a randomly oriented sample of sources we obtain $\theta=44^{\circ}$ for $0.4<z<0.6$, while for $z<0.3$ we have $\theta=37^{\circ}$.

However, according to the new picture presented here, in Paper II, and in agreement with the previous findings of Laing et al. (1994), it is plausible that LEG constitute a separate sample, and should not be included in the statistics of the unification scheme for FR II and quasars. In this case, we obtain $\theta=49^{\circ}$ for $0.4<z<0.6$ and $\theta=43^{\circ}$ or $46^{\circ}$ for 
$z<0.3$, considering all the unclassified objects as HEG or LEG, respectively.

Barthel et al. (1989) found $\theta=44^{\circ}$ as the dividing angle between QSO and radio galaxies for the sample of 3CR sources with $0.5<z<1$. However, if we re-classify the sources in the light of their nuclear properties and considering the most recent spectral information ${ }^{1}$, we obtain that in the Barthel sample $44 \%$ of the objects are BLO, 53\% are HEG and only $2 \%$ (corresponding to 1 source) are LEG. This corresponds to a torus opening angle of $56^{\circ}$. Therefore, regardless of the shape of the obscuring structure, this is a clear indication for a decreasing covering factor with increasing redshift.

Willott et al. (2000) found that the quasar fraction (and thus the torus opening angle) in low-frequency-selected samples of radio sources increases as luminosity and redshift increase. They proposed that the statistical properties of their samples could be explained by the receding torus model (Lawrence 1991). In this scenario, the internal radius of the obscuring dusty torus increases as the luminosity of the central AGN increases, since dust sublimates at a fixed temperature $(T \sim 1500 \mathrm{~K})$. As an alternative, they pointed out that the emergence of a population of low- $z$ FR I-like "starved quasars" can also account for their findings.

By classifying the objects according to their nuclear properties, and thus considering the LEG as a separate population, we still find that the opening angle increases with redshift. Therefore, having excluded the majority of the FR I-like population, we favor the receding torus model as the most plausible scenario to account for the statistics of FR II and quasars.

\section{Conclusions}

We have analyzed the optical emission from the nuclei of 26 FR II radio sources within the redshift range $0.4<z<0.6$. Our results are consistent with the behavior observed in lower redshift objects. Although in this range of redshifts the identification of CCC is not straightforward, $\sim 50 \%$ of the sources show an unresolved nuclear component.

The behavior of the sources in the plane formed by the optical core luminosity plotted vs. the radio core luminosity is related to their optical spectral classification. Objects with broad lines show an optical excess with respect to the correlation found for FR I objects. This excess is most plausibly due to thermal emission from the accretion disk.

Only one LEG of our sample shows a CCC; it lies on the FR I correlation. The upper limits for the other two LEG are also compatible with the correlation. This is essentially the same behavior as observed in low- $z$ objects.

The two HEG which have a CCC, as well as the five optical upper limits, are consistent with the FR I correlation (within $2 \sigma$ ). As for low- $z$ objects, the nature of their nuclei is classified by the inclusion of a further parameter, i.e. the luminosity of the $[\mathrm{OIII}]$ emission line. The location of the nuclei in the plane formed by the equivalent width of the [OIII] emission line vs. the optical-to-radio core flux ratio indicates whether we

\footnotetext{
${ }^{1}$ http://www-astro.physics.ox.ac.uk/cjw/3crr/ $3 \mathrm{crr} . \mathrm{html}$
}

are directly observing the nuclear ionizing continuum or if the source of ionization is absorbed to our line-of-sight.

BLO are found in the same region as their low- $z$ counterparts, therefore we interpret them as unabsorbed nuclei. The HEG lie in the upper part of the plane, indicating that we are observing only a fraction of their ionizing continuum source. 3C 244.1 has both high $E W([\mathrm{OIII}])$ and optical excess, therefore we propose that this source is a moderately absorbed BLO or, alternatively, a HEG with a scattered nucleus and with a rather unusual optical-to-radio core flux ratio. Future deep spectroscopic observations can test this hypothesis.

In the FR II-QSO unification scenario the number ratio of BLO to narrow-line objects has been used to statistically estimate the opening angle of the obscuring torus. In the light of our optical studies and considering the most recent spectral information we confirm that the opening angle increases with redshift. The most plausible explanation is provided by the receding torus model (e.g., Lawrence 1991).

Acknowledgements. The authors thank William B. Sparks and Gabriele Giovannini for useful discussions. This research has made use of the NASA/IPAC Extragalactic Database (NED) which is operated by the Jet Propulsion Laboratory, California Institute of Technology, Under contract with the National Aeronautics and Space Administration. This work has been partially supported by the Summer Student Program (SSP) at the Space Telescope Science Institute and by the Institute of Radioastronomy.

\section{References}

Baggett, S., et al. 2002, in HST WFPC2 Data Handbook, v. 4.0, ed. B. Mobasher, Baltimore, STScI

Barthel, P. D. 1989, ApJ, 336, 606

Baum, S. A., Heckman, T. M., Bridle, A., et al. 1988, ApJS, 68, 643

Bogers, W. J., Hes, R., Barthel, P. D., \& Zensus, J. A. 1994, A\&AS, 105, 91

Chiaberge, M., Capetti, A., \& Celotti, A. 1999, A\&A, 349, 77

Chiaberge, M., Capetti, A., \& Celotti, A. 2000, A\&A, 355, 873

Chiaberge, M., Capetti, A., \& Celotti, A. 2002, A\&A, 394, 791

De Koff, S., Baum, S. A., Sparks, W. B., et al. 1996, 107, 621

Eracleous, M., \& Halpern, J. P. 1994, ApJ, 433, L17

Fanaroff, B. L., \& Riley, J. M. 1974, MNRAS, 167, 31

Hardcastle, M. J. 2004, A\&A, 414, 927

Jackson, C. A., \& Wall, J. V. 1999, MNRAS, 304, 160

Jackson, N., \& Rawlings, S. 1997, MNRAS, 286, 241

Jackson, N., \& Browne, I. W. A. 1991, MNRAS, 250, 414

Laing, R. A., Jenkins, C. R., Wall, J. V., \& Unger, S. W. 1994, The First Stromlo Symposium: The Physics of Active Galaxies, ed. G. V. Bicknell, M. A. Dopita, \& P. J. Quinn, ASP Conf. Ser., 54, 201

Lawrence, A. 1991, MNRAS, 252, 586

Lehnert, M. D., Miley, G. K., Sparks, W. B., et al. 1999, ApJS, 123, 351

Martel, A. R., Baum, S. A., Sparks, W. B., et al. 1999, ApJS, 122, 81

Moore, R. L., \& Stockman, H. S. 1981, ApJ, 243, 60

Spinrad, H., Marr, J., Aguilar, L., \& Djorgovski, S. 1985, PASP, 97, 932

Tadhunter, C. N., Morganti, R., di Serego Alighieri, S., Fosbury, R. A. E., \& Danziger, I. J. 1993, MNRAS, 263, 999

Urry, C. M., \& Padovani, P. 1995, PASP, 107, 803

Willott, C. J., Rawlings, S., Blundell, K. M., \& Lacy, M. 2000, MNRAS, 316, 449

Zirbel, E. L. 1996, ApJ, 473, 713

Zirbel, E. L. 1997, ApJ, 476, 489 